\title{
A COMMUTATIVE NOETHERIAN HOPF ALGEBRA OVER A FIELD IS FINITELY GENERATED
}

\author{
RICHARD K. MOLNAR
}

ABSTRACT. Let $k$ be an arbitrary field and $H$ a commutative Hopf algebra over $k$. We give a short proof of the fact that $H$ is Noetherian if and only if $H$ is finitely generated as a $k$-algebra.

In [4] M. Takeuchi has shown that a commutative (or cocommutative) Hopf algebra $H$ is faithfully flat over any sub-Hopf algebra $K$. He then uses this result to give a simple proof of the following:

(INJ) If $H$ is a commutative Hopf algebra over a field $k$ then the correspondence

$$
\begin{aligned}
\text { (sub-Hopf algebras) } & \rightarrow \text { (Hopf ideals) } \\
K & \mapsto K^{+} H
\end{aligned}
$$

is injective.

As a corollary to (INJ) Takeuchi shows $[4,3.11]$ that any sub-Hopf algebra of a finitely generated, commutative Hopf algebra over a field is finitely generated. The purpose of this note is to show that a commutative Noetherian Hopf algebra over a field is finitely generated, and that this fact follows from (INJ) with essentially the same proof as Takeuchi's corollary.

For any Hopf algebra $H$ over the field $k$, we let $H^{+}=\operatorname{ker}(\epsilon)$ where $\epsilon$ is the counit of $H$, and we denote the antipode of $H$ by $S$. A commutative Hopf algebra is said to be Noetherian (resp. finitely generated) if it is Noetherian (resp. finitely generated) as a $k$-algebra. For the basic facts about Hopf algebras see [1] or [3]. Clearly the desired result follows from the following

Proposition. Let $H$ be a commutative Hopf algebra over a field $k$. If $H^{+}$is finitely generated as an ideal then $H$ is finitely generated.

Proof. Let $\left\{f_{1}, \ldots, f_{n}\right\}$ be generators of the ideal $H^{+}$. If $C$ is the subcoalgebra of $H$ generated by the $f_{i}$, then $C$ is finite dimensional [1,

Received by the editors December 2, 1974 and, in revised form, April 1, 1975. AMS (MOS) subject classifications (1970). Primary 16A24; Secondary 13E05. Key words and phrases. Commutative Hopf algebra, Noetherian algebra. 
1.4.3]. Since the antipode of $H$ has order 2 , it follows that $D=C+S(C)$ is a finite dimensional subcoalgebra of $H$ which is stable under the antipode, and $\left\{f_{1}, \ldots, f_{n}\right\} \subset D$. If we let $[D]$ be the subalgebra of $H$ generated by $D$, then, in fact, $[D]$ is a sub-Hopf algebra of $H$ which is finitely generated.

Now $\left\{f_{1}, \ldots, f_{n}\right\} \subset[D] \cap H^{+}=[D]^{+}$, and since the $f_{i}$ are generators of the ideal $H^{+}$, we must have $H^{+} \subset[D]^{+} H$. On the other hand, $[D]^{+} H$ is clearly contained in $H^{+}$. Thus $[D]^{+} H=H^{+}=H^{+} H$, and so by (INJ) we must have $[D]=H$, which gives the desired conclusion.

One may easily generalize this result. For example, by using $\mathrm{K}$. Newman's version of (INJ) for cocommutative Hopf algebras $[2,2.5]^{1}$ along with the proof of the Proposition (which essentially depends only on (INJ) and the fact that the antipode has finite order), one can show that a cocommutative Noetherian Hopf algebra over a field is finitely generated.

\section{REFERENCES}

1. R. G. Heyneman and M. E. Sweedler, Affine Hopf algebras. I, J. Algebra 13 (1969), 192-241. MR 39 \#6876.

2. K. Newman, A correspondence between bi-ideals and sub-Hopf algebras in cocommutative Hopf algebras (preprint).

3. M. E. Sweedler, Hopf algebras, Math. Lecture Note Series, Benjamin, New York, 1969. MR 40 \#5705.

4. M. Takeuchi, A correspondence between Hopf ideals and sub-Hopf algebras, Manuscripta Math. 7 (1972), 251-270. MR 48 \#328. 48063

DEPARTMENT OF MATHEMATICS, OAKLAND UNIVERSITY, ROCHESTER, MICHIGAN

1 We thank the referee for pointing this out. 